This item was submitted to Loughborough's Research Repository by the author.

Items in Figshare are protected by copyright, with all rights reserved, unless otherwise indicated.

\title{
Dynamic decision making in lane change: game theory with receding horizon
}

PLEASE CITE THE PUBLISHED VERSION

https://dx.doi.org/10.1109/CONTROL.2016.7737643

PUBLISHER

(c) IEEE

VERSION

AM (Accepted Manuscript)

LICENCE

CC BY-NC-ND 4.0

REPOSITORY RECORD

Meng, Fanlin, Jinya Su, Cunjia Liu, and Wen-Hua Chen. 2016. "Dynamic Decision Making in Lane Change:

Game Theory with Receding Horizon". figshare. https://hdl.handle.net/2134/21900. 


\title{
Dynamic Decision Making in Lane Change: Game Theory with Receding Horizon
}

\author{
Fanlin Meng, Jinya Su, Cunjia Liu, and Wen-Hua Chen \\ Department of Aeronautical and Automotive Engineering \\ Loughborough LE11 3TU, U.K. \\ \{F.Meng, J.Su2, C.Liu5, W.Chen\}@ lboro.ac.uk
}

\begin{abstract}
Decision making for lane change manoeuvre is of practical importance to guarantee a smooth, efficient and safe operation for autonomous driving. It is, however, challenging. On one hand, the behaviours of ego vehicle and adjacent vehicles are dependent and interactive. On the other hand, the decision should strictly guarantee safety during the whole process of lane change with uncertain and incomplete information in a dynamic and cluttered environment. To this end, the concept of Receding Horizon Control (RHC) is integrated into game theory in conjunction with reachability analysis tool, resulting in RHC based game theory. Specifically, the decision of each game relies on not only uncertain information at current step but also the future information calculated by reachability analysis. The decision is repeatedly made with the advent of new information using the concept of RHC. As a result, safety can be guaranteed during the whole process of lane change in a dynamic environment. Case study is conducted to demonstrate the advantages of the proposed approach. It is shown that the proposed RHC based game theory approach incorporating uncertain information can provide a safer and real-time decision.
\end{abstract}

Index Terms-Game theory, Lane change, analysis, Receding horizon, Safety assessment

\section{INTRODUCTION}

Lane-change manoeuvre, as one of the most important and commonly encountered automatic driving operations for autonomous vehicles, is receiving increasing attention in both academia and industry recently [1]. On one hand, the lanechange manoeuvre is a necessity for performing other more complicated operations such as leaving the road, overtaking another vehicle among others [2]. On the other hand, this manoeuvre is a major source of congestion and collisions [3]. Among many lane-change indexes (such as efficiency, comfort), safety is the highest priority, which must be strictly guaranteed in the whole process of lane change within an uncertain dynamic environment.

Conventional lane change models (e.g., Gipps Model [4] and MOBIL [5]) only assume one-direction impact of surrounding vehicles on the ego vehicle. This assumption may not be true in practice since the ego vehicle can also affect the decision of surrounding vehicles [6]. As a result, the lane change decision problem involves multiple vehicles interacting with each other. Game theory provides a promising framework for scenarios where interaction is involved [3] [6] [7]. Kita [6] pioneered the work of applying game theory to lane change decision in mandatory merging scenario, where the information about surrounding vehicles such as their velocities and distances is assumed to be available. Recently, Vehicle-to-Vehicle (V2V) communications is used in [3] to improve drivers' awareness about surrounding traffic conditions and consequently lead to a safer and more efficient driving manoeuvre.

In this paper, however, no coordination (via V2V or vehicle to central station communication) is assumed, whereas the information about surrounding vehicles purely relies on the on-board sensors. This poses new research challenges. First, the information of surrounding vehicles at current step (e.g., position, velocity) inferred through filtering algorithms using on-board sensors is inevitably subject to errors due to many factors. For example, forward-looking radar devices for vehicle tracking may result in unsatisfying tracking accuracy due to low angular resolution; vision sensors are often vulnerable to poor weather and lighting conditions. This leads to uncertainties at current step.

Secondly, the future information of the surrounding vehicles is important for safety assessment in lane change decision. The commonly used approach is to predict vehicles' future information several seconds ahead based on the inferred information at current step. If perfect information about surrounding vehicles at current step and the kinematics model are known, trajectory prediction could be realized by a simple mathematical calculation [8]. However, as pointed out in [9], this assumption is not realistic in real environments. Apart from the uncertainties at current step, the physical model describing the vehicle movement is subject to uncertainties (e.g., driver's intentional or unintentional manoeuvre). This leads to uncertainties in the prediction model.

To account for the aforementioned uncertainties such that safety can be strictly guaranteed [10], uncertain interval models are adopted in this paper to capture the uncertainness at current step (i.e., position, velocity) and uncertainties in the prediction models (i.e., drivers' uncertain manoeuvre). On this basis, reachability analysis in [11] can be drawn to calculate all possible trajectories of the surrounding vehicles in future time horizon. Worst case analysis is explicitly derived such that the upper and lower bound of all possible trajectories can be determined in a more computation-efficient way. This is possible by exploiting the special structure of the reachability analysis problem under consideration, where no uncertainties appear in the system matrices. The calculated bound information is then used in the pay-off matrix calculation involved in the game theory model. 
Furthermore, the surrounding environment for the ego vehicle is dynamically changing. As a result, a decision made at current step may be obsolete for the next several steps. Consequently, it is more useful and rational to repeatedly make decision with the advent of new information rather than implementing one fixed decision for the rest of steps. This strategy coincides with the idea of Receding Horizon Control (RHC) from control engineering [12]. In RHC, the decision is derived by repeatedly solving a constrained optimization problem over a moving $N$-step-ahead horizon based on the information at current step [13], where only the first action is applied to the system. At the next step, the time horizon is shifted one step forward and the same optimizing procedure for another $N$ steps in the near future is repeatedly solved with new information.

Consequently, in this paper, the concept of RHC is integrated into game theory in conjunction with reachability analysis tool. At each step, the decision is made for the interactive players using game theory maximizing their mutual payoffs, where the uncertainties in the surrounding vehicles are effectively handled by reachability analysis. Then the concept of RHC is applied such that the decision is repeatedly made with the advent of new information. Simulation study is conducted, which shows that the proposed decision making strategy taking uncertainties into account can guarantee a safer decision in comparison with the one without using uncertain information. At the same time, it is also shown that by using the concept of RHC, an updated decision can be provided at each step with the advent of new information.

\section{Problem Statement AND PRELiminaries}

\section{A. Problem Statement}

There are two types of lane changes, i.e., Mandatory Lane Change (MLC) and Discretionary Lane Change (DLC). MLC occurs when a driver must change lane to follow a specified path due to lane closure ahead, while DLC occurs when a driver changes to a lane perceived to obtain better traffic conditions [1]. In this paper, DLC is considered.

Figure 1 shows a scenario of DLC by ego vehicle $M$. In this scenario, the speed of vehicle $M$ is lower than its desired speed, which is limited by its leading vehicle $L_{a}$ on Lane $A$ and vehicle $M$ plans to change from Lane A to Lane B to get a better driving condition, e.g., closer to its desired speed. However, the following questions should be addressed : 1) Is it safe to make the lane change? 2) Is it worth making the lane change?

Motivated by the observations from practical traffic situations where the ego vehicle can also affect the decisions of surrounding vehicles, we adopt a game theory approach to model the lane change decision problem which involves multiple vehicles interacting with each other.

The lane change game for the scenario in Figure 1 consists of two players, i.e., the ego vehicle $M$ and the following vehicle $F_{b}$. Each vehicle makes decision by taking into account the other vehicles' potential response and consequently the game belongs to a two-player non-cooperative game.

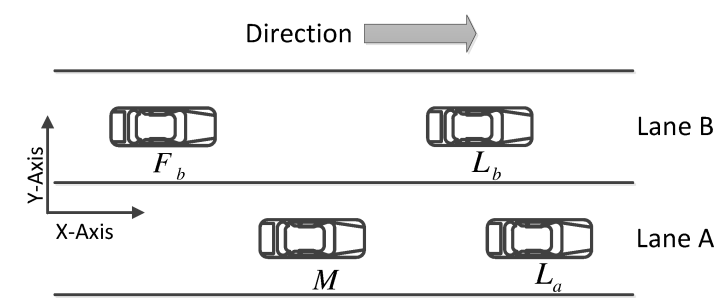

Fig. 1: Discretionary Lane Change Scenario.

\section{B. Non-cooperative Game}

In a non-cooperative game, each player makes decisions independently. Nash equilibrium (NE) is a solution concept of the non-cooperative game involving two or more players, in which no player has anything to gain by changing only their own strategy [14].

Let $(S, f)$ be a game with $n$ players, where $S_{i}$ is the strategy set for player $i, S=S_{1} \times S_{2} \times \cdots \times S_{n}$ is the set of strategy profiles and $f(x)=\left(f_{1}(x), \ldots, f_{n}(x)\right)$ is the pay-off function evaluated at strategy profile $x \in S$. Let $x_{i} \in S_{i}$ be the strategy of player $i$ and $x_{-i}$ be the strategies of all players except player $i$. When each player $i \in\{1, \ldots, n\}$ chooses strategy $x_{i}$ resulting in a strategy profile $x=\left(x_{1}, \ldots, x_{n}\right)$, player $i$ obtains pay-off $f_{i}(x)$. A strategy profile $x^{*} \in S$ is a Nash equilibrium if no unilateral deviation in strategy by any single player is profitable for that player [15] [16], that is:

$$
\forall i, x_{i} \in S_{i}: f_{i}\left(x_{i}^{*}, x_{-i}^{*}\right) \geq f_{i}\left(x_{i}, x_{-i}^{*}\right) .
$$

\section{Reachability analysis}

Reachability analysis can potentially calculate all possible trajectories of a given uncertain system with initial state/parameter/input uncertainties [11]. Consider an uncertain system described by interval matrices

$$
\dot{x} \in \mathcal{A} x+\mathcal{B} u,
$$

where $x$ is the uncertain state vector with uncertain initial value $x(0) \in X_{0}$ and $u \in U$ is the uncertain system input, interval matrix $\mathcal{A}=[\underline{A}, \bar{A}]$ and $\mathcal{B}=[\underline{B}, \bar{B}]$ are the uncertain system and input matrices respectively, with $\underline{A}, \underline{B}$ and $\bar{A}, \bar{B}$ being their lower bound and upper bound matrices.

The exact state reachable sets $R^{e}(r)$ for a given time $t=r$ is formally defined as

$$
\begin{aligned}
R^{e}(r)= & \left\{x(r) \mid x(t)=\int_{0}^{t}[A x(\tau)+B u(\tau)] d \tau,\right. \\
& \left.x(0) \in X_{0}, A \in \mathcal{A}, B \in \mathcal{B}, \forall t: u \in U\right\} .
\end{aligned}
$$

Although exact reachable set computation can only be achieved for a limited class of systems, tools are available to over-approximate the reachable set in a tight way (see [11] and [17] among many others). It is a promising tool to conduct safety assessment considering the uncertain characteristics and safety-critical requirements in autonomous driving.

The point-mass kinematic equations of motion describing vehicle motion in the longitudinal direction [18] is given by

$$
\underbrace{\left[\begin{array}{c}
\dot{s} \\
\dot{v}
\end{array}\right]}_{\dot{x}}=\underbrace{\left[\begin{array}{ll}
0 & 1 \\
0 & 0
\end{array}\right]}_{A} x+\underbrace{\left[\begin{array}{l}
0 \\
1
\end{array}\right]}_{B} u,
$$


where $s, v$ denote its position and velocity. Suppose that the uncertain intervals are given by $s(0) \in\left[s(0)_{\min }, s(0)_{\max }\right]$ with $s(0)_{w i d}=s(0)_{\max }-s(0)_{\min }$ denoting its width, $v(0) \in$ $\left[v(0)_{\min }, v(0)_{\max }\right]$ with $v(0)_{w i d}=v(0)_{\max }-v(0)_{\min }$ and $u \in\left[u_{\min }, u_{\max }\right]$ with $u_{w i d}=u_{\max }-u_{\min }$. As a result, Eq. (2) falls into the form of system (1) and the reachability analysis tool can be applied.

However, considering the special structure of Eq. (2) where uncertainties only appear in the input in an additive way and initial states rather than system matrices, the following simple mathematical manipulation can provide a more efficient way to calculate the upper and lower bound of the uncertain variables of interest. The solution of Eq. (2) at step $t$ is given by

$$
x(t)=e^{A t} x(0)+\int_{0}^{t} e^{A(t-\tau)} B u(\tau) d \tau, e^{A t}=\left[\begin{array}{ll}
1 & t \\
0 & 1
\end{array}\right] .
$$

from which, one can obtain $s(t)$ and $v(t)$, given by

$$
\left\{\begin{array}{l}
s(t)=s(0)+t v(0)+0.5 t^{2} u \\
v(t)=v(0) t+t u
\end{array} .\right.
$$

It follows from Eq. (3) that for all $t \geq 0, s(t)$ is a monotonic increasing function of $s(0), v(0)$ and $u$. As such, the upper and lower bounds of $s(t)$ are given by

$$
\left\{\begin{array}{l}
s(t)_{\min }=s(0)_{\min }+t v(0)_{\min }+0.5 t^{2} u_{\min } \\
s(t)_{\max }=s(0)_{\max }+t v(0)_{\max }+0.5 t^{2} u_{\max }
\end{array} .\right.
$$

\section{OUR Proposed APPROACH}

\section{A. Lane Change Game Formulation}

In our proposed lane change game, the interactions between the ego vehicle $M$ and the following vehicle $F_{b}$ are considered. We will first discuss the strategies of each player and then formulate their pay-off matrix.

Following [3] [6], we consider two strategies for the ego vehicle (i.e., change lane (CL) and not change lane (NCL)) and following vehicle (i.e., acceleration (AC) and deceleration (DE)). Deceleration can be seen as a courtesy yielding by the following vehicle while acceleration can be understood as not willing to give way. The pay-off matrix of this lane change game can be formulated as Table I

TABLE I: Pay-off matrix of the proposed lane change game

\begin{tabular}{|l|c|c|}
\hline Vehicle $M$ & $A C$ & $D E$ \\
\hline$C L$ & $E 11, F 11$ & $E 12, F 12$ \\
\hline$N C L$ & $E 21, F 21$ & $E 22, F 22$ \\
\hline
\end{tabular}

\section{B. Vehicle Information at time $t_{0}$ and $t_{T}$}

1) Vehicle Information at $t_{0}$ and Assumptions: At decision time $t_{0}$, we assume the initial information of vehicles are available as follows:

- The speed of vehicle $M$ is $v_{M}^{0}$ and position of $M$ is $s_{M}^{0}$;

- The speed of vehicle $L_{a}$ is $v_{L_{a}}^{0}$ and position of $L_{a}$ is $s_{L_{a}}^{0}$;

- The speed of vehicle $L_{b}$ is $v_{L_{b}}^{0}$ and position of $L_{b}$ is $s_{L_{b}}^{0}$;

- The speed of vehicle $F_{b}$ is $v_{F_{b}}^{0}$ and position of $F_{b}$ is $s_{F_{b}}^{0}$.
Assume vehicle $M$ needs a duration of time $T$ to finish the lane change manoeuvre. We denote $t_{T}=t_{0}+T$.

When making lane change decisions for vehicle $M$ at time $t_{0}$, the future information (position/speed at $t_{T}$ ) of all the vehicles are needed to be taken into account.

It is further assumed that vehicles $M, L_{a}$ and $L_{b}$ are at constant speed in this study.

Assume that if vehicle $F_{b}$ accelerates, it will choose a preferred acceleration of $a_{0}^{a c}$ which is only known to $F_{b}$ itself. However, the ego vehicle $M$ cannot get this exact acceleration information of $F_{b}$. The acceleration of $F_{b}$ perceived by the ego vehicle can be represented as $a^{a c} \in\left[a_{\text {min }}^{a c}, a_{\text {max }}^{a c}\right]$; Similarly, if $F_{b}$ decelerates, it will choose a preferred deceleration of $a_{0}^{d e}$ which is only known to itself and the deceleration of $F_{b}$ perceived by the ego vehicle is $a^{d e} \in\left[a_{\text {min }}^{d e}, a_{\text {max }}^{d e}\right]$.

At time $t_{0}$, vehicle $M$ can get the velocity information of $F_{b}$ as $v_{F_{b}}^{\prime 0} \in\left[v_{\text {min }}^{0}, v_{\text {max }}^{0}\right]$ and the position as $s_{F_{b}}^{\prime 0} \in\left[s_{\min }^{0}, s_{\max }^{0}\right]$ via on-board sensors.

2) Estimated Vehicle Information at $t_{T}$ : Based on above vehicle information at time $t_{0}$ in conjunction with the reachability analysis tool discussed in Section II-C, we have the upper bound and lower bound of the position of vehicle $F_{b}$ at time $t_{T}$ perceived by vehicle $M$ computed as follows, where Eq. (4) is for acceleration while Eq. (5) is for deceleration.

$$
\begin{gathered}
\left\{\begin{array}{l}
s_{\text {max }}^{a c}=s_{\text {max }}^{0}+v_{\text {max }}^{0}+\frac{1}{2} a_{\text {max }}^{a c} T^{2} \\
s_{\text {min }}^{a c}=s_{\text {min }}^{0}+v_{\text {min }}^{0}+\frac{1}{2} a_{\text {min }}^{a c} T^{2}
\end{array},\right. \\
\left\{\begin{array}{l}
s_{\max }^{d e}=s_{\text {max }}^{0}+v_{\text {max }}^{0}+\frac{1}{2} a_{\text {max }}^{d e} T^{2} \\
s_{\text {min }}^{d e}=s_{\text {min }}^{0}+v_{\text {min }}^{0}+\frac{1}{2} a_{\text {min }}^{d e} T^{2}
\end{array} .\right.
\end{gathered}
$$

Furthermore, positions of vehicles $M, L_{a}, L_{b}$ at time $t_{T}$ are given as follows:

$$
\left\{\begin{array}{l}
s_{M}^{T}=s_{M}^{0}+v_{M}^{0} \times T \\
s_{L_{a}}^{T}=s_{L_{a}}^{0}+v_{L_{a}}^{0} \times T \\
s_{L_{b}}^{T}=s_{L_{b}}^{0}+v_{L_{b}}^{0} \times T
\end{array},\right.
$$

while positions of $F_{b}$ at time $t_{T}$ under its preferred acceleration $a_{0}^{a c}$ and deceleration $a_{0}^{d e}$, which are only accurately known to $F_{b}$ itself, are given by

$$
\left\{\begin{array}{c}
s_{a c}^{T}=s_{F_{b}}^{0}+v_{F_{b}}^{0} \times T+\frac{1}{2} a_{0}^{a c} \times T^{2} \\
s_{d e}^{T}=s_{F_{b}}^{0}+v_{F_{b}}^{0} \times T+\frac{1}{2} a_{0}^{d e} \times T^{2}
\end{array} .\right.
$$

\section{Pay-off Quantification for Ego Vehicle $M$}

We define the length of the vehicles as $L$. The minimum safe gap between $M$ and $F_{b}$ for safe lane change is $G_{F_{b}}$, while the minimum safe gap between $L_{b}$ and $M$ for safe lane change is $G_{L_{b}}$. Both the safety and the potential speed gain of the ego vehicle are considered in quantifying its payoff.

Firstly, we quantify the payoffs of ego vehicle $M$ if it changes lane based on Rule 1 and Rule 2. Depending on how the following vehicle $F_{b}$ acts, there are two payoffs for $M$. If $F_{b}$ accelerates, the payoff of $M$ is $E 11$; if $F_{b}$ decelerates, the payoff of $M$ is $E 12$. If it is safe for the ego vehicle $M$ to make a lane change (i.e., the gaps between the ego vehicle and other related vehicles at time $t_{T}$ are no less than any of 
the minimum safe gaps), the payoffs of $M$ are then quantified by its potential speed gain which is represented as the velocity difference between the leading vehicle on the target lane (i.e., $L_{b}$ ) and the leading vehicle on the current lane (i.e., $L_{a}$ ). Otherwise, a high penalty (-50 in this study) is applied.

- Rule 1: Rule for quantifying E11

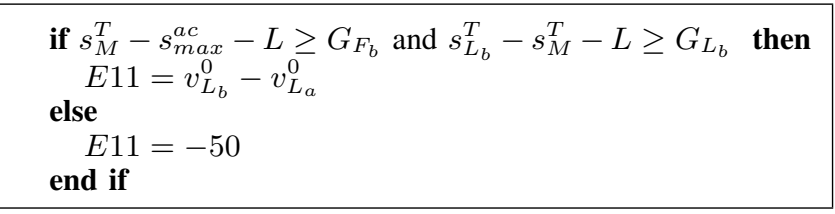

- Rule 2: Rule for quantifying $E 12$

$$
\begin{aligned}
& \text { if } s_{M}^{T}-s_{\max }^{d e}-L \geq G_{F_{b}} \text { and } s_{L_{b}}^{T}-s_{M}^{T}-L \geq G_{L_{b}} \text { then } \\
& \quad E 12=v_{L_{b}}^{0}-v_{L_{a}}^{0} \\
& \text { else } \\
& \quad E 12=-50 \\
& \text { end if }
\end{aligned}
$$

Secondly, we quantify the payoffs of ego vehicle $M$ if it does not change lane. As the driving condition of $M$ in this case does not change and is not affected by the potential actions of vehicle $F_{b}$, we set the payoffs of $M$ (i.e., $E 21$, $E 22)$ to zero. That is,

$$
\text { - } E 21=0
$$$$
\text { - } E 22=0 \text {. }
$$

\section{Pay-off Quantification for Following Vehicle $F_{b}$}

For following vehicle $F_{b}$, we consider safety and speed variation in quantifying its pay-off. Note that if vehicle $M$ changes lane, the gap between $F_{b}$ and $M$ at time $t_{T}$ will be checked first to guarantee the driving safety of $F_{b}$. If vehicle $M$ does not change lane, the gap between $F_{b}$ and $L_{b}$ will be checked instead to guarantee the driving safety of $F_{b}$.

It is further assumed that the higher the speed variation of $F_{b}$, the lower the payoff. The above assumption is reasonable due to the fact that a higher speed variation will cause more inconvenience or disturbance to the driving conditions of the vehicle and therefore result in a lower payoff. In this study, the speed variation is defined as acceleration or the absolute value of deceleration of the following vehicle $F_{b}$.

Firstly, we quantify the payoffs of following vehicle $F_{b}$ based on Rule 3 and Rule 4 if it accelerates. Depending on how the ego vehicle $M$ acts, there are two payoffs for $F_{b}$. If $M$ changes lane, the payoff of $F_{b}$ is $F 11$; if $M$ does not change lane, the payoff of $F_{b}$ is $F 21$. If the driving safety of $F_{b}$ is guaranteed, the payoffs of $F_{b}$ are quantified by its speed variation which is represented as the reciprocal of its preferred acceleration. Otherwise, a high penalty (-50 in this study) is applied.

- Rule 3: Rule for quantifying $F 11$

$$
\begin{aligned}
& \text { if } s_{M}^{T}-s_{a c}^{T}-L \geq G_{F_{b}} \text { then } \\
& \quad F 11=\frac{1}{a_{0}^{a c}} \\
& \text { else } \\
& \quad F 11=-50 \\
& \text { end if }
\end{aligned}
$$

- Rule 4: Rule for quantifying $F 21$

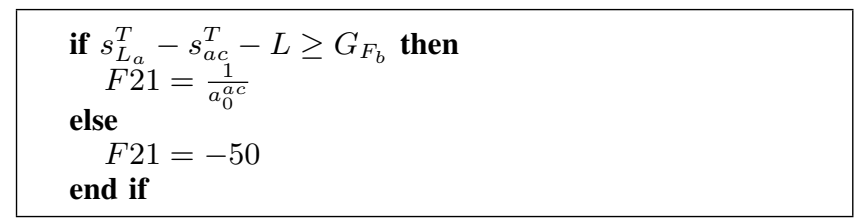

Secondly, we quantify the payoffs of following vehicle $F_{b}$ according to Rule 5 and Rule 6 if it decelerates.

- Rule 5: Rule for quantifying $F 12$

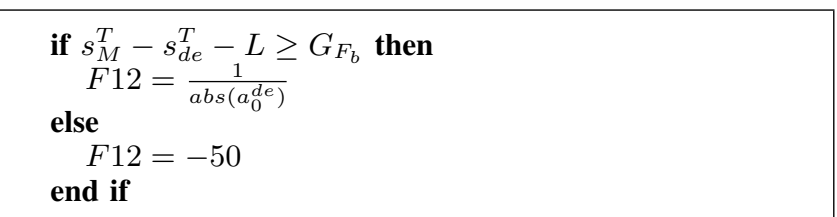

- Rule 6: Rule for quantifying $F 22$

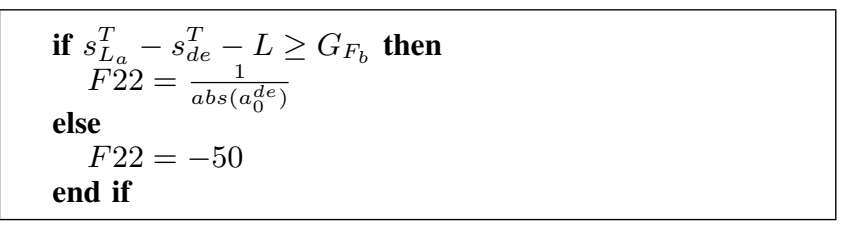

where $a b s\left(a_{0}^{d e}\right)$ represents the absolution value of the deceleration rate $a_{0}^{d e}$.

\section{E. Lane Change Game Solution}

The solution concept of this lane change game is Nash equilibrium (NE). We consider solving pure strategy NE for this game, where all players are playing pure strategies, i.e., each player chooses one strategy from its strategy set with a probability of one [16].

According to the definition of Nash equilibrium [14], a strategy profile is a Nash equilibrium only if each strategy in that strategy profile is a best response to all the other strategies in that same strategy profile. Following the above definition, a pure strategy Nash equilibrium for this lane change game can be analytically solved [19].

\section{CASE Study}

In the section, case studies are conducted to demonstrate the advantages of the proposed lane change game model where the payoffs of players are quantified using reachability analysis. The vehicle information at time $t_{0}$ as well as some other parameters used in the simulation are given in Table II

\section{A. Decision at current step $t_{0}$}

First, we compare the game behaviours of vehicle $M$ under two different cases. The first case happens when reachability analysis is used by ego vehicle $M$ to estimate the future position information of $F_{d}$ at time $t_{T}$ and to quantify the game payoff matrix accordingly. On the other hand, the second case assumes that vehicle $M$ uses perceived point values other than intervals to estimate the position of $F_{d}$.

For the second case, we assume the perceived speed of vehicle $F_{d}$ by vehicle $M$ at time $t_{0}$ is $15.5 \mathrm{~m} / \mathrm{s}$, the perceived 
TABLE II: Parameter Settings for Lane Change Game Simulations

\begin{tabular}{|c|c|c|}
\hline Parameter & Description & Setting Value \\
\hline$L$ & Length of the vehicle & $3.5 \mathrm{~m}$ \\
\hline$T$ & Lane change time duration & $4 s$ \\
\hline$v_{L_{\alpha}}^{0}$ & Constant speed of vehicle $L_{a}$ & $25 \mathrm{~m} / \mathrm{s}$ \\
\hline$s_{L_{a}}^{0}$ & position of vehicle $L_{a}$ at time $t_{0}$ & $60 \mathrm{~m}$ \\
\hline$v_{L_{b}}^{0^{a}}$ & Constant speed of vehicle $L_{b}$ & $30 \mathrm{~m} / \mathrm{s}$ \\
\hline$s_{L_{b}}^{0}$ & position of vehicle $L_{b}$ at time $t_{0}$ & $55 \mathrm{~m}$ \\
\hline$v_{M}^{0}$ & Constant speed of vehicle $M$ & $17 \mathrm{~m} / \mathrm{s}$ \\
\hline$s_{M}^{0}$ & position of vehicle $M$ at time $t_{0}$ & $\begin{array}{l}40 \mathrm{~m} \\
15 \mathrm{~m} / \mathrm{s}\end{array}$ \\
\hline$v_{F_{b}}$ & $\begin{array}{l}\text { Initial speed of vehicle } F_{b} \text { at time } t_{0} \text {, } \\
\text { only known to vehicle } F_{b}\end{array}$ & $15 \mathrm{~m} / \mathrm{s}$ \\
\hline$s_{F_{b}}^{0}$ & $\begin{array}{l}\text { Position of vehicle } F_{b} \text { at time } t_{0} \text {, only } \\
\text { known to vehicle } F_{b}\end{array}$ & $20 \mathrm{~m}$ \\
\hline$a_{0}^{a c}$ & $\begin{array}{l}\text { Acceleration used by vehicle } F_{b} \text { if it } \\
\text { accelerates }\end{array}$ & $1.2 \mathrm{~m} / \mathrm{s}^{2}$ \\
\hline$a_{0}^{d e}$ & $\begin{array}{l}\text { Deceleration used by vehicle } F_{b} \text { if it } \\
\text { decelerates }\end{array}$ & $-1.5 \mathrm{~m} / \mathrm{s}^{2}$ \\
\hline$v_{F_{b}}^{\prime 0}$ & $\begin{array}{l}\text { Speed interval of vehicle } F_{b} \text { at time } t_{0} \\
\text { estimated by the on-board sensors of } \\
\text { vehicle } M\end{array}$ & {$[15,16] \mathrm{m} / \mathrm{s}$} \\
\hline$s_{F_{b}}^{\prime 0}$ & $\begin{array}{l}\text { Position interval of vehicle } F_{b} \text { at time } \\
t_{0} \text { estimated by the on-board sensors } \\
\text { of vehicle } M\end{array}$ & {$[20,22.5] m$} \\
\hline$a^{a c}$ & $\begin{array}{l}\text { Acceleration interval of } F_{d} \text { perceived } \\
\text { by ego vehicle } M\end{array}$ & {$[1,2] \mathrm{m} / \mathrm{s}^{2}$} \\
\hline$a^{d e}$ & $\begin{array}{l}\text { Deceleration interval of } F_{d} \text { perceived } \\
\text { by ego vehicle } M\end{array}$ & {$[-2,-1] m / s^{2}$} \\
\hline$G_{F_{b}}$ & $\begin{array}{l}\text { Minimum safe gap between vehicle } M \\
\text { and } F_{b} \text { if } M \text { changes lane }\end{array}$ & $3 \times L$ \\
\hline$G_{L_{b}}$ & $\begin{array}{l}\text { Minimum safe gap between vehicle } L_{b} \\
\text { and } M \text { if } M \text { changes lane }\end{array}$ & $3 \times L$ \\
\hline
\end{tabular}

TABLE III: Pay-off matrix under first case at current step $t_{0}$, interval based estimation of vehicle $F_{d}$ via reachability analysis

\begin{tabular}{|l|c|c|}
\hline Vehicle $M$ & $A C$ & $D E$ \\
\hline$C L$ & $-50,0.83$ & $5,0.66$ \\
\hline$N C L$ & $\underline{\mathbf{0}, \underline{\mathbf{0 . 3 3}}}$ & $0,0.66$ \\
\hline
\end{tabular}

TABLE IV: Pay-off matrix under second case at current step $t_{0}$, point based estimation of vehicle $F_{d}$

\begin{tabular}{|c|c|c|}
\hline $\begin{array}{lll}\text { Vehicle } M & \text { Vehicle } F_{b}\end{array}$ & $A C$ & $D E$ \\
\hline$C L$ & $\underline{5}, \underline{0.83}$ & $5,0.66$ \\
\hline$N C L$ & $\overline{0.83}$ & $0,0.66$ \\
\hline
\end{tabular}

position of $F_{d}$ at time $t_{0}$ is $21.25 \mathrm{~m}$, the perceived acceleration of $F_{d}$ at time $t_{0}$ is $1.2 \mathrm{~m} / \mathrm{s}^{2}$ and the perceived deceleration of $F_{d}$ at time $t_{0}$ is $-1 \mathrm{~m} / \mathrm{s}^{2}$.

Given above parameters settings, by using our proposed approach, the lane change game matrices and the resulting Nash Equilibria (underlined) are given in Tables III and IV

One can see from Tables III and IV that in the first case, the decision of ego vehicle $M$ is not to change lane, while the decision of $M$ under the second case is to change lane.

These different game behaviours of $M$ under the above two cases (note that vehicle $F_{b}$ is accelerating in both cases) can be better understood by looking into Figure 2 where the position of the ego vehicle from time $t_{0}$ to $t_{T}$ and the perceived positions of the following vehicle under both the point based estimation and the interval based estimation from time $t_{0}$ to

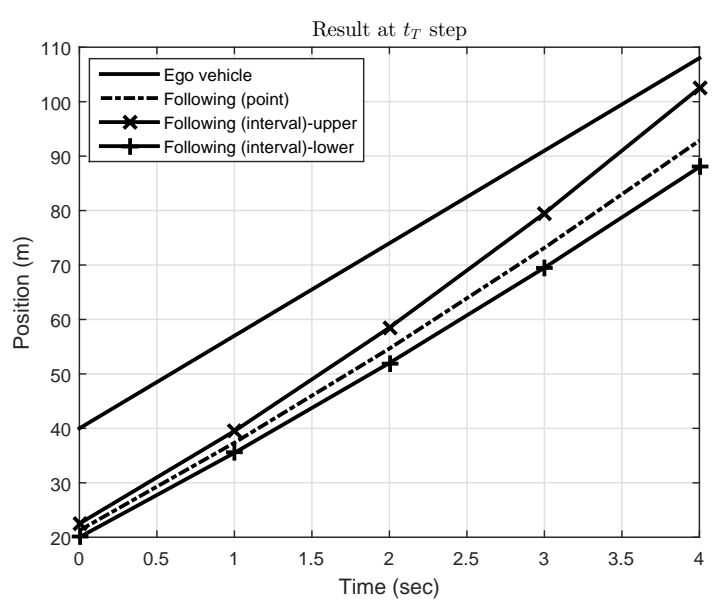

Fig. 2: The predicted position of ego vehicle and following vehicle (following vehicle accelerates) based on information at time $t_{0}$ for a prediction horizon of time $T=4$ seconds.

$t_{T}$ are illustrated in Figure 2

As aforementioned, since vehicle $M$ always tends to avoid collision with other vehicles and guarantee its safety in any situation, it will use worst case analysis when faced with uncertainties in decision making. As a result, under the first case, vehicle $M$ uses the perceived upper bound position information of the following vehicle $F_{b}$ to decide if it should change lane or not. As one can see from Figure 2 , the distance between ego vehicle $M$ and the following vehicle under the first case at the $4^{\text {th }}$ second is less than the minimum gap $G_{F_{b}}$. To guarantee its own safety, $M$ will choose not to change lane. This leads to a Nash equilibrium of $(N C L, A C)$. On the contrary, the distance between $M$ and $F_{d}$ under second case is greater than the minimum safe gap $G_{F_{b}}$, which leads to a Nash equilibrium of $(C L, A C)$.

In other words, the decision made via our proposed reachability analysis based game approach by taking the future uncertain information of surrounding vehicles into account is safer and can avoid any potential collisions in the lane change process.

\section{B. Decision at next time step $t_{1}$}

At the next time step, new information about the surrounding vehicles might be available. Consequently, following the idea of RHC, a new decision might need to be made by taking the new information into account. We suppose at time $t_{1}$ (i.e., the next time step), the newly measured position information about the vehicle $F_{b}$ is available, given by [28, 30.2] $m$ (the rest information of $F_{b}$ remains the same, and all the information about other vehicles is just shifted one step ahead based on Table II). The lane change game matrix and the resulting Nash Equilibria (underlined) at time $t_{1}$ are given in Tables $\mathrm{V}$ and $\mathrm{VI}$ respectively.

For both the point based estimation case and the interval based estimation case, the decision of the ego vehicle $M$ is to change lane. As one can see from Figure 3 the distance 
TABLE V: Pay-off matrix under first case at $t_{1}$, interval based estimation of vehicle $F_{d}$ via reachability analysis

\begin{tabular}{|c|c|c|}
\hline $\begin{array}{ll}\text { Vehicle } M & \text { Vehicle } F_{b}\end{array}$ & $A C$ & $D E$ \\
\hline$C L$ & $\underline{5}, \underline{0.83}$ & $5,0.66$ \\
\hline$N C L$ & $\overline{0.83}$ & $0,0.66$ \\
\hline
\end{tabular}

TABLE VI: Pay-off matrix under second case at $t_{1}$, point based estimation of vehicle $F_{d}$

\begin{tabular}{|c|c|c|}
\hline $\begin{array}{ll}\text { Vehicle } M & \text { Vehicle } F_{b}\end{array}$ & $A C$ & $D E$ \\
\hline$C L$ & $5,0.83$ & $5,0.66$ \\
\hline$N C L$ & $\overline{0,0.83}$ & $0,0.66$ \\
\hline
\end{tabular}

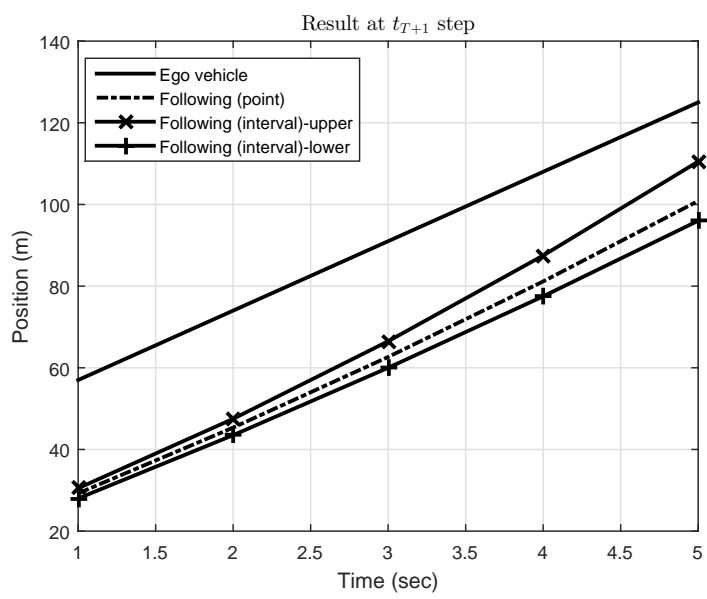

Fig. 3: The predicted position of ego vehicle and following vehicle (following vehicle accelerates) based on new information at time $t_{1}$ for a prediction horizon of time $T=4$ seconds.

between the ego vehicle and the following vehicle under both cases at the $5^{\text {th }}$ second is higher than the minimum gap $G_{F_{b}}$. This case study demonstrates that by using the concept of RHC, the decision can be changed/updated by taking new information into account. This is of particular importance for a safe and smooth lane change in a dynamic uncertain environment.

\section{CONCLUSiOnS AND FUTURE WORK}

This paper proposed a safer and dynamic decision making strategy for lane change manoeuvre of autonomous vehicles using game theory. Considering the uncertain information of surrounding vehicles, reachability analysis is first drawn to calculate all the possible trajectories of surrounding vehicles, which is then used in the payoff calculation of game theory. The concept of Receding Horizon Control (RHC) is integrated into game theory such that the decision is repeatedly made with the advent of new information. As a result, safety can be strictly guaranteed during the whole process of lane change manoeuvre under dynamic uncertain environments. Comparison case study is conducted to demonstrate the advantages of the proposed approach. It is shown that the proposed RHC based game theory approach incorporating uncertain information provides a safer and real-time decision. Future work will be done to reduce the uncertainties by learning the behaviour (e.g., driving styles) of surrounding vehicles using machine learning techniques so as to have a better understanding of the intentions of other drivers.

\section{ACKNOWLEDGEMENT}

This work is jointly supported by the UK Engineering and Physical Sciences Research Council (EPSRC) Autonomous and Intelligent Systems programme under the grant number $\mathrm{EP} / \mathrm{J} 011525 / 1$ with BAE Systems as the leading industrial partner.

\section{REFERENCES}

[1] K. I. Ahmed, Modeling drivers' acceleration and lane changing behavior. PhD thesis, Massachusetts Institute of Technology, 1999.

[2] J. E. Naranjo, C. Gonzalez, R. Garcia, and T. De Pedro, "Lane-change fuzzy control in autonomous vehicles for the overtaking maneuver," Intelligent Transportation Systems, IEEE Transactions on, vol. 9, no. 3, pp. 438-450, 2008.

[3] A. Talebpour, H. S. Mahmassani, and S. H. Hamdar, "Modeling lane-changing behavior in a connected environment: A game theory approach," Transportation Research Part C: Emerging Technologies, vol. 59, pp. 216-232, 2015.

[4] P. G. Gipps, "A model for the structure of lane-changing decisions," Transportation Research Part B: Methodological, vol. 20, no. 5, pp. 403414, 1986.

[5] A. Kesting, M. Treiber, and D. Helbing, "General lane-changing model mobil for car-following models," Transportation Research Record: Journal of the Transportation Research Board, 2007.

[6] H. Kita, "A merging-giveway interaction model of cars in a merging section: a game theoretic analysis," Transportation Research Part A: Policy and Practice, vol. 33, no. 3, pp. 305-312, 1999.

[7] R. Elvik, "A review of game-theoretic models of road user behaviour," Accident Analysis \& Prevention, vol. 62, pp. 388-396, 2014.

[8] R. Miller and Q. Huang, "An adaptive peer-to-peer collision warning system," in Vehicular technology conference, 2002. VTC Spring 2002. IEEE 55th, vol. 1, pp. 317-321, IEEE, 2002.

[9] D. Ferguson, M. Darms, C. Urmson, and S. Kolski, "Detection, prediction, and avoidance of dynamic obstacles in urban environments," in Intelligent Vehicles Symposium, 2008 IEEE, pp. 1149-1154, IEEE, 2008.

[10] S. Arora, A. Raina, and A. Mittal, "Collision avoidance among agvs at junctions," in Intelligent Vehicles Symposium, 2000. IV 2000. Proceedings of the IEEE, pp. 585-589, IEEE, 2000.

[11] M. Althoff, O. Stursberg, and M. Buss, "Reachability analysis of linear systems with uncertain parameters and inputs," in Decision and Control, 2007 46th IEEE Conference on, pp. 726-732, IEEE, 2007.

[12] D. W. Clarke, "Advances in model-based predictive control," 1994.

[13] X.-B. Hu and W.-H. Chen, "Receding horizon control for aircraft arrival sequencing and scheduling," Intelligent Transportation Systems, IEEE Transactions on, vol. 6, no. 2, pp. 189-197, 2005.

[14] J. Nash, "Non-cooperative games," Annals of mathematics, pp. 286-295, 1951.

[15] Wikipedia, "Nash equilibrium - wikipedia, the free encyclopedia," 2016. [Online; accessed 20-February-2016].

[16] M. J. Osborne and A. Rubinstein, A course in game theory. MIT press, 1994.

[17] J. Su and W.-H. Chen, "Fault diagnosis for vehicle lateral dynamics with robust threshold," in IEEE International Conference on Industrial Technology, Accepted, 2016.

[18] M. Jalalmaab, B. Fidan, S. Jeon, and P. Falcone, "Model predictive path planning with time-varying safety constraints for highway autonomous driving," in Advanced Robotics (ICAR), 2015 International Conference on, pp. 213-217, IEEE, 2015.

[19] R. W. Rosenthal, "A class of games possessing pure-strategy nash equilibria," International Journal of Game Theory, vol. 2, no. 1, pp. 6567, 1973. 